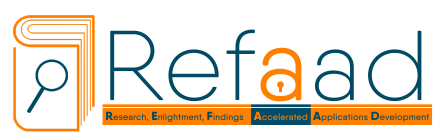

General Letters in Mathematics Vol. 2, No. 2, April 2017, pp.73-83

e-ISSN 2519-9277, p-ISSN 2519-9269

Available online at http:// www.refaad.com

\title{
Existence of Solution of Neutral Fractional Impulsive Differential Equations with Infinite Delay
}

\author{
K.Hilal* and Y. Allaoui ${ }^{2}$ \\ ${ }^{12}$ Laboratoire de Mathématique Appliquées \& Calcul Scientifique \\ Sultan Moulay Slimane University, BP 523, 23000 Béni Mellal, Morocco \\ ${ }^{1}$ hilal2005@yahoo.fr, ${ }^{2}$ youssefbenlarbi1990@gmail.com
}

\begin{abstract}
In this work we define the solution of the fractional neutral impulsive differential equation with infinite delay. The results of existence are obtained by using the Banach contraction and Schafer fixed point. Some proprieties of probability density functions and semi-group theory are also used.
\end{abstract}

Keywords: Fixed point theorem, Fractional impulsive differential equations, Neutral equations, infinite delay.

\section{Introduction}

Differential equation of fractional order occur more frequently different research areas and engineering, such as physics, biology, chemistry, economics, control of dynamical systems, etc. Numerous books and articles focused in this field, see the monographs Podlubny [11, Miller and Ross [10, Kilbas [6] and papers [12, [8], [9], [5], [7].

The class of impulsive differential functional equations have attracted much attention where the impulsion is instantaneous. It has modeled many physical phenomena processes in the real world, it is often subjected to abrupt changes such as shocks, and short time perturbations during the evolution of processes, so it attracted many authors [3], [2], [5].

In [3] the authors studied the existence of solutions for an abstract impulsive fractional differential equations where the impulses are not instantaneous is that the subject of some dynamics of evolution processes in Pharmacotherapy. In fact this situation of impulsives actions starts at an arbitray fixed point and stays active on a finite time interval, and the model equations reads

$$
\left\{\begin{array}{l}
D^{\alpha} x(t)=A x(t)+f(t, x(t)), t \in\left(s_{i}, t_{i+1}\right], i=0,1,2 \ldots, m, \\
x(t)=I_{i}\left(x\left(t_{i}\right)\right)+g_{i}(t, x(t)), t \in\left(t_{i}, s_{i}\right], i=1,2, \ldots, m, \\
x(0)=x_{0},
\end{array}\right.
$$

where $A: D(A) \subset X \longrightarrow X$ is the generator of $C_{0}$-semigroup of bounded linear operators $\{T(t)\}_{t \geq 0}$ on Banach space $(X,\|\cdot\|), 0=t_{0}=s_{0}<t_{1} \leq s_{1}<t_{2} \ldots \leq s_{m}<t_{m+1}=T$ are fixed numbers, $g_{i} \in C\left(\left(t_{i}, s_{i}\right] \times X, X\right)$ and $I_{i}: X \longrightarrow X$, for $(i=1,2, \ldots, m)$. At the point $s_{i}$, the function $x$ is continuous. The term $I_{i}\left(x\left(t_{i}\right)\right)$ means that the impulses are related to the value of $x\left(t_{i}\right)=x\left(t_{i}^{-}\right)$.

${ }^{*}$ Corresponding author.K.Hilal ${ }^{1}$ hilal2005@yahoo.fr 2010 Mathematics Subject Classification: 58C30, 34K45, 34A08, 34K40.. 
Motivated by [1] where the authors studied the following problem

$$
\left\{\begin{array}{l}
D^{\alpha} x(t)=f\left(t, x_{t}\right), \text { for each } t \in J=[0, T], 0<\alpha<1, \\
x(t)=\phi(t), \quad t \in(-\infty, 0]
\end{array}\right.
$$

where $f: J \times \mathfrak{B} \rightarrow \mathbb{R}, \mathfrak{B}$ the phase space. For any function $x$ defined on $(-\infty, T]$ and $t \in J$, we denote by $x_{t}$ the element of $\mathfrak{B}$ defined by

$$
x_{t}(\theta)=x(t+\theta), \theta \in(-\infty, 0] .
$$

We shall study the following problem:

$$
\text { (1) }\left\{\begin{array}{l}
D^{\alpha}\left(x(t)-h\left(t, x_{t}\right)\right)=A\left(x(t)-h\left(t, x_{t}\right)\right)+f\left(t, x_{t}\right), \quad t \in\left(s_{i}, t_{i+1}\right], i=0,1,2 \ldots, m \\
x(t)=I_{i}\left(x\left(t_{i}\right)\right)+g_{i}(t, x(t)), t \in\left(t_{i}, s_{i}\right] \quad i=1,2, \ldots, m \\
x_{0}=\phi \in \mathfrak{B}
\end{array}\right.
$$

where $A, g_{i}, I_{i}$ which are already given, and $f: J \times \mathfrak{B} \rightarrow X$ and $h: J \times \mathfrak{B} \rightarrow X$ are appropriate functions to be specified later, and $D^{\alpha}$ is the Caputo fractional derivative with $0<\alpha<1$.

The outline of this paper goes as follows. In section 2 we introduce some preliminaries about fractional calculus, and about construction of solution involving probability density function. In section 3 we establish the existence of solution to the problem (1) by Banach's contraction and schafer's fixed point.

\section{Preliminary Notes}

In this section, we introduce definitions and results which will be used throughout this paper.

Definition 2.1. [12] Let $x \in C^{n}[0, \infty)$ and $n-1<\alpha<n$, the Caputo's derivative of order $\alpha$ for function $x:[0, \infty) \longrightarrow \mathbb{R}$ can be written as

$$
D^{\alpha} x(t)=\frac{1}{\Gamma(n-\alpha)} \int_{0}^{t}(t-s)^{n-\alpha-1} x^{(n)}(s) d s .
$$

Definition 2.2. [12] The fractional integral of order $\alpha$ is defined as

$$
I^{\alpha} x(t)=\frac{1}{\Gamma(\alpha)} \int_{0}^{t}(t-s)^{\alpha-1} x(s) d s
$$

which is called the Riemann-Liouville integral.

To study the fractional differential equation with infinite delay, we assume that the space $\left(\mathfrak{B},\|\cdot\|_{\mathfrak{B}}\right)$ is a seminormed linear space of functions mapping $(-\infty, 0]$ into $\mathbb{R}$, and satisfying the following fundamental axioms introduced by Hale and Kato in [4].

(A) If $x:(-\infty, T] \rightarrow \mathbb{R}$, and $y_{0} \in \mathfrak{B}$, then for every $t \in[0, T]$ the following conditions hold:

i) $x_{t}$ is in $\mathfrak{B}$,

ii) $\left\|x_{t}\right\|_{\mathfrak{B}} \leq C_{1}(t) \sup \{|x(s)|: 0 \leq s \leq t\}+C_{2}(t)\left\|x_{0}\right\|_{\mathfrak{B}}$, where $C_{1}:[0, T] \rightarrow[0, \infty)$ is continuous, $C_{2}:[0, \infty) \rightarrow$ $[0, \infty)$ is locally bounded and $C_{1}, C_{2}$ are independent of $x($.$) .$

$(A-1)$ For the function $x($.$) in (A), x_{t}$ is a $\mathfrak{B}$-valued continuous function on $[0, T]$.

$(A-2)$ The space $\mathfrak{B}$ is complete. 
We consider the space $\mathfrak{B}^{\prime}=\left\{x:(-\infty, T] \longrightarrow X\right.$ such that $x \in C\left(J_{i}, X\right)$ and there exist $x\left(t_{i}^{-}\right)$and $x\left(t_{i}^{+}\right)$, $i=1,2, \ldots, m$ with $\left.x\left(t_{k}\right)=x\left(t_{i}^{-}\right), x_{0}=\phi \in \mathfrak{B}\right\}$, where $J=[0, T]$, and $J_{i}=\left(t_{i}, t_{i+1}\right]$, for $i=1, \ldots, m$, and we define the semi-norm in $\mathfrak{B}^{\prime}$ by

$$
\|x\|_{\mathfrak{B}^{\prime}}=\sup _{t \in J}\|x(t)\|+\|\phi\|_{\mathfrak{B}}, \quad x \in \mathfrak{B}^{\prime} .
$$

Lemma 2.3. [2] Let $v:[0, T] \longrightarrow[0, \infty)$ be a real function, $\omega$ a nonnegative and locally integrable function on $[0, T]$, and there are constant $a>0$, and $0<\alpha<1$ such that

$$
v(t) \leq \omega(t)+a \int_{0}^{t} \frac{v(s)}{(t-s)^{\alpha}} d s,
$$

then there exists a constant $K(\alpha)$ such that

$$
v(t) \leq w(t)+a K(\alpha) \int_{0}^{t} \frac{w(s)}{(t-s)^{\alpha}} d s .
$$

Lemma 2.4. [Q] Let $P$ be a continuous and compact mapping on Banach space $X$ into itself, such that the set $\{x \in X: x=\lambda P x$, for some $0<\lambda<1\}$ is bounded, then $P$ has a fixed point.

Consider the following problem

$$
(2)\left\{\begin{array}{l}
D^{\alpha}\left(x(t)-h\left(t, x_{t}\right)\right)=A\left(x(t)-h\left(t, x_{t}\right)\right)+f(t), \quad t \in\left(s_{i}, t_{i+1}\right], i=0,1,2 \ldots, m, \\
x(t)=I_{i}\left(x\left(t_{i}\right)\right)+g_{i}(t, x(t)), t \in\left(t_{i}, s_{i}\right] \quad i=1,2, \ldots, m, \\
x_{0}=\phi \in \mathfrak{B} .
\end{array}\right.
$$

To construct the solution for this problem we follow these steps :

- Step 1: We apply the operator $I^{\alpha}$ to (the first equation of the) problem (2) we obtain

$$
\left\{\begin{aligned}
& x(t)-h\left(t, x_{t}\right)=\left(\phi(0)-h\left(0, x_{0}\right)\right) \\
&+\frac{1}{\Gamma(\alpha)} \int_{0}^{t}(t-s)^{\alpha-1} A\left(x(s)-h\left(s, x_{s}\right)\right)+f(s) d s, t \in\left[0, t_{1}\right], \\
& x(t)=I_{1}\left(x\left(t_{1}\right)\right)+g_{1}(t, x(t)), t \in\left(t_{1}, s_{1}\right] \\
& x(t)-h\left(t, x_{t}\right)=d_{i}+\frac{1}{\Gamma(\alpha)} \int_{0}^{t}(t-s)^{\alpha-1} A\left(x(s)-h\left(s, x_{s}\right)\right)+f(s) d s, t \in\left[s_{1}, t_{2}\right] \\
& \vdots \\
& x(t)=I_{i}\left(x\left(t_{i}\right)\right)+g_{i}(t, x(t)), t \in\left(t_{i}, s_{i}\right] \\
& x(t)-h\left(t, x_{t}\right)=d_{i}+\frac{1}{\Gamma(\alpha)} \int_{0}^{t}(t-s)^{\alpha-1} A\left(x(s)-h\left(s, x_{s}\right)\right)+f(s) d s, t \in\left[s_{i}, t_{i+1}\right]
\end{aligned}\right.
$$

for $i=1,2, \ldots, m$, and $d_{i} \in X$. The continuity property of the function $x$ at points $s_{i}$ allows us to write

$$
x(t)-h\left(t, x_{t}\right)=d_{i} \chi_{\left[s_{i}, t_{i+1}\right)}+\frac{1}{\Gamma(\alpha)} \int_{0}^{t}(t-s)^{\alpha-1} A\left(x(s)-h\left(s, x_{s}\right)\right)+f(s) d s, \quad t \in\left(s_{i}, t_{i+1}\right],
$$

for $i=1,2, \ldots, m$, and where $\chi_{\left[s_{i}, t_{i+1}\right)}(t)$ is the characteristic function of $\left[s_{i}, t_{i+1}\right)$.

- Step 2: We apply the Laplace transformations and we follow the idea used in [13] to obtain $\mathfrak{u}(\lambda)=\lambda^{\alpha-1}\left(\lambda^{\alpha} I-A\right)^{-1} e^{-\lambda s_{i}} d_{i}-\lambda^{\alpha-1}\left(\lambda^{\alpha} I-A\right)^{-1} e^{-\lambda t_{i+1}} d_{i}+\left(\lambda^{\alpha} I-A\right)^{-1} \mathrm{v}(\lambda)$, 
where $\mathfrak{u}$ and $\boldsymbol{v}$ are Laplace transformation of the function $x-h$ and $f$ and $I$ is the identity operator defined in $X$.

We follow the same computation in [12, 13, and using inverse Laplace transformation and the property that

$$
\int_{0}^{\infty} e^{-\lambda s} \omega_{\alpha}(s) d s=e^{-\lambda^{\alpha}}
$$

is Laplace transformation of $\omega_{\alpha}$ given in [12, 13] by

$$
\omega_{\alpha}(s)=\frac{1}{\pi} \sum_{n=1}^{\infty}(-1)^{n-1} s^{-n \alpha-1} \frac{\Gamma(n \alpha+1)}{n !} \sin (n \pi \alpha), \quad s \in(0, \infty),
$$

We conclude that

$$
x(t)-h\left(t, x_{t}\right)=P_{\alpha}\left(t-s_{i}\right) d_{i} \chi_{\left[s_{i}, t_{i+1}\right)}+\int_{0}^{t}(t-s)^{\alpha-1} Q_{\alpha}(t-s) f(s) d s, t \in\left(s_{i}, t_{i+1}\right],
$$

where

$$
P_{\alpha}(t)=\int_{0}^{\infty} \xi_{\alpha}(s) T\left(t^{\alpha} s\right) d s
$$

and

$$
Q_{\alpha}(t)=\alpha \int_{0}^{\infty} s \xi_{\alpha}(s) T\left(t^{\alpha} s\right) d s
$$

with

$$
\begin{gathered}
\xi_{\alpha}(s)=\frac{1}{\alpha} s^{-1-\frac{1}{\alpha}} \omega_{\alpha}\left(s^{-\frac{1}{\alpha}}\right), \\
\int_{0}^{\infty} \xi_{\alpha}(s) d s=1, \\
\int_{0}^{\infty} s \xi_{\alpha}(s) d s=\frac{1}{\Gamma(\alpha+1)},
\end{gathered}
$$

where the relation (1), 22) and (3) are given in 12,13 .

- Step 3: We determine the values of $d_{i}$ using the fact that $x$ is continuous at the points $s_{i}$. We get

$$
d_{i}=I_{i}\left(x\left(t_{i}\right)\right)-h\left(s_{i}, x_{s_{i}}\right)+g_{i}\left(s_{i}, x\left(s_{i}\right)\right)-\int_{0}^{s_{i}}\left(s_{i}-s\right)^{\alpha-1} Q_{\alpha}\left(s_{i}-s\right) f(s) d s .
$$

Now we are in a position to define our solution.

Definition 2.5. The function $x \in \mathfrak{B}^{\prime}$ is mild solution of problem (1) if it satisfies

$$
(7) x(t)=\left\{\begin{array}{l}
\phi(t), t \in(-\infty, 0], \\
P_{\alpha}(t)\left(\phi(0)-h\left(0, x_{0}\right)\right)+h\left(t, x_{t}\right)+\int_{0}^{t}(t-s)^{\alpha-1} Q_{\alpha}(t-s) f\left(s, x_{s}\right) d s, t \in\left[0, t_{1}\right] \\
I_{i}\left(x\left(t_{i}\right)\right)+g_{i}(t, x(t)), t \in\left(t_{i}, s_{i}\right] \\
\vdots \\
P_{\alpha}(t) d_{i}+h\left(t, x_{t}\right)+\int_{0}^{t}(t-s)^{\alpha-1} Q_{\alpha}(t-s) f\left(s, x_{s}\right) d s, \quad t \in\left[s_{i}, t_{i+1}\right] .
\end{array}\right.
$$

We make the following assumption on $A$ in the whole paper.

$H(A)$ The operator $A$ is generator of a strongly continuous semigroup $\{T(t): t \geq 0\}$ in $X$, and $\sup _{t \in[0, \infty)} \|$ $T(t) \|_{L(X)} \leq M_{A}$ with $M_{A} \geq 1$. For $t>0, T(t)$ is compact. 
Lemma 2.6. 3] Let $H(A)$ hold then the operators $P_{\alpha}$ and $Q_{\alpha}$ have the following properties:

1. For any fixed $t \geq 0 P_{\alpha}(t), Q_{\alpha}(t)$ are linear and bounded operators, and for any $x \in X$

$$
\left\|P_{\alpha}(t) x\right\| \leq M_{A}\|x\|,\left\|Q_{\alpha}(t) x\right\| \leq \frac{\alpha M_{A}}{\Gamma(\alpha+1)}\|x\| .
$$

2. $\left\{P_{\alpha}(t), t \geq 0\right\}$ and $\left\{Q_{\alpha}(t), t \geq 0\right\}$ are strongly continuous.

3. for every $t>0 P_{\alpha}(t)$ and $Q_{\alpha}(t)$ are compact operators.

\section{Main results}

This section will be devoted to the study of the existence of the solution of problem (1). In order to do this, we make the following assumptions:

(H1) $f:[0, T] \times \mathfrak{B} \rightarrow X$ is continuous, and there exists $L_{f}$ such that

$$
\|f(t, \varphi)-f(t, \psi)\| \leq L_{f}\|\varphi-\psi\|_{\mathfrak{B}} . \quad \text { for } t \in J, \varphi, \psi \in \mathfrak{B},
$$

$h:[0, T] \times \mathfrak{B} \rightarrow X$ is continuous, and there exists $L_{h}$ such that

$$
\left\|h\left(t_{1}, \varphi\right)-h\left(t_{2}, \psi\right)\right\| \leq L_{h}\left(\left|t_{1}-t_{2}\right|+\|\varphi-\psi\|_{\mathfrak{B}}\right) .
$$

(H2) $C_{1}=\sup _{t \in[0, T]} C_{1}(t), \quad C_{2}=\sup _{t \in[0, T]} C_{2}(t)$,

(H3) There exists $L_{I}$, such that $\left\|I_{i}(x)-I_{i}(y)\right\| \leq L_{I}\|x-y\|, i=1,2, \ldots, m$, for $x, y \in X$.

There exists $L_{g}$ such that $\left\|g_{i}(t, x)-g_{i}(t, y)\right\| \leq L_{g}\|x-y\|, i=1,2, \ldots, m$, for $x, y \in X$.

The first result is based on Banach contraction.

Theorem 3.1. Assume that $(A),(H 1),(H 2)$ and (H3) are satisfied. Furthermore

$$
\left(M_{A}\left(L_{I}+L_{g}+C_{1} L_{h}+\frac{M_{A} C_{1} L_{f}}{\Gamma(\alpha+1)} T^{\alpha}\right)+\frac{C_{1} M_{A}}{\Gamma(\alpha+1)} T^{\alpha} C_{1} L_{f}+C_{1} L_{h}\right)<1 .
$$

Then, the problem (1) has a unique solution.

Proof. Let the operator $N: \mathfrak{B}^{\prime} \longrightarrow \mathfrak{B}^{\prime}$ defined by

$$
N(x)(t)=\left\{\begin{aligned}
& \phi(t), t \in(-\infty, 0] \\
& P_{\alpha}(t)(\phi(0)-\left.h\left(0, x_{0}\right)\right)+h\left(t, x_{t}\right) \\
& \quad+\int_{0}^{t}(t-s)^{\alpha-1} Q_{\alpha}(t-s) f\left(s, x_{s}\right) d s, t \in\left[0, t_{1}\right] \\
& I_{1}\left(x\left(t_{1}\right)\right)+g_{1}(t, x(t)), t \in\left(t_{1}, s_{1}\right] \\
& \vdots \\
& I_{i}\left(x\left(t_{i}\right)\right)+g_{i}(t, x(t)), t \in\left(t_{i}, s_{i}\right] \\
& P_{\alpha}(t) d_{i}+h\left(t, x_{t}\right)+\int_{0}^{t}(t-s)^{\alpha-1} Q_{\alpha}(t-s) f\left(s, x_{s}\right) d s, t \in\left[s_{i}, t_{i+1}\right]
\end{aligned}\right.
$$

Let $y():.(-\infty, T] \longrightarrow X$ be the function defined by

$$
y(t)=\left\{\begin{array}{l}
0 \text { if } t \in[0, T] \\
\phi(t) \text { if } t \in(-\infty, 0]
\end{array}\right.
$$


Then, $y_{0}=\phi$. For each $z \in C([0, T], X)$ with $z(0)=0$, we denote by $\bar{z}$ the function defined by

$$
\bar{z}(t)=\left\{\begin{array}{l}
z(t) \text { if } t \in[0, T] \\
0 \text { if } t \in(-\infty, 0]
\end{array}\right.
$$

So we decompose $x($.$) as x(t)=y(t)+\bar{z}(t)$ for $t \in[0, T]$, which implies $x_{t}=y_{t}+\bar{z}_{t}$ for $t \in[0, T]$ where $x($.$) satisfies$ the relation (7), the function $z($.$) is given by$

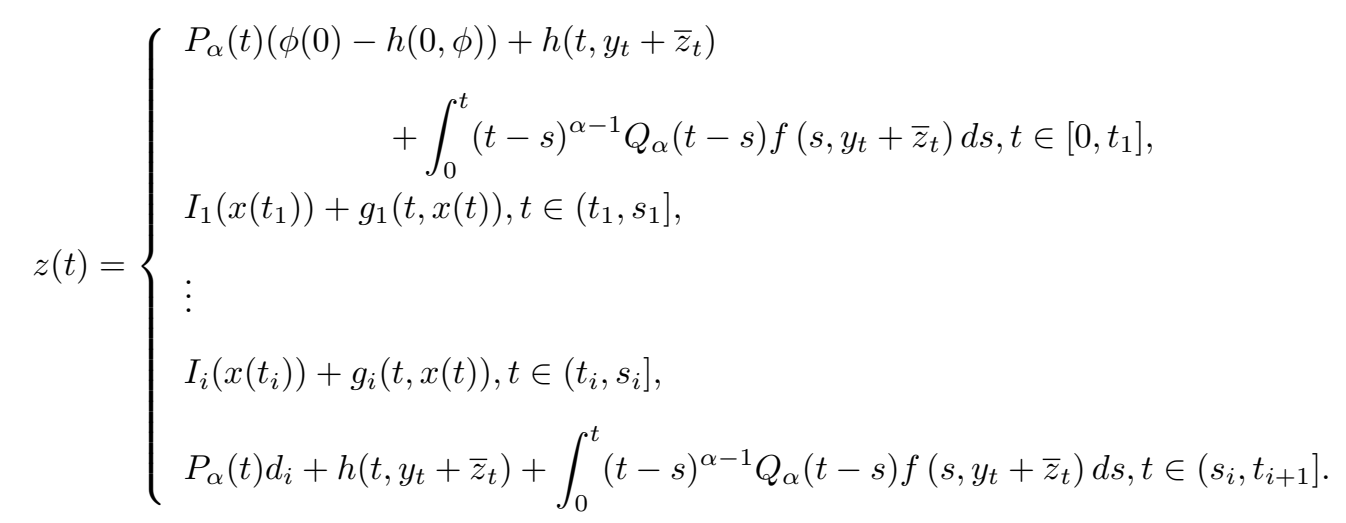

Set

$$
\mathfrak{B}^{\prime \prime}=\left\{z \in \mathfrak{B}^{\prime} \text { such that } z_{0}=0\right\}
$$

and let $\|\cdot\|_{\mathfrak{B}^{\prime \prime}}$ be the seminorm in $\mathfrak{B}^{\prime \prime}$ defined by

$$
\|z\|_{\mathfrak{B}^{\prime \prime}}=\sup _{t \in J}\|z(t)\|+\left\|z_{0}\right\|_{\mathfrak{B}}=\sup _{t \in J}\|z(t)\|, \quad z \in \mathfrak{B}^{\prime \prime}
$$

$\mathfrak{B}^{\prime \prime}$ is a Banach space with norm $\|\cdot\|_{\mathfrak{B}^{\prime \prime}}$.

We define the operator $P: \mathfrak{B}^{\prime \prime} \longrightarrow \mathfrak{B}^{\prime \prime}$ by

$$
P(z)(t)=\left\{\begin{aligned}
& P_{\alpha}(t)(\phi(0)-h(0, \phi))+h\left(t, y_{t}+\bar{z}_{t}\right) \\
& \quad+\int_{0}^{t}(t-s)^{\alpha-1} Q_{\alpha}(t-s) f\left(s, y_{t}+\bar{z}_{t}\right) d s, \quad t \in\left[0, t_{1}\right], \\
& I_{1}\left(x\left(t_{1}\right)\right)+g_{1}(t, x(t)), t \in\left(t_{1}, s_{1}\right] \\
& \vdots \\
& I_{i}\left(x\left(t_{i}\right)\right)+g_{i}(t, x(t)), t \in\left(t_{i}, s_{i}\right] \\
& P_{\alpha}(t) d_{i}+h\left(t, y_{t}+\bar{z}_{t}\right)+\int_{0}^{t}(t-s)^{\alpha-1} Q_{\alpha}(t-s) f\left(s, y_{t}+\bar{z}_{t}\right) d s, t \in\left(s_{i}, t_{i+1}\right] .
\end{aligned}\right.
$$

Note that the operator $N$ has a unique fixed point is equivalent to $P$ has a unique fixed point too. Let us prove that 
$P$ has a fixed point. Consider $z, z^{*} \in \mathfrak{B}^{\prime \prime}$, then we have for $t \in\left[0, t_{1}\right]$

$$
\begin{aligned}
\left\|P(z)(t)-P\left(z^{*}\right)(t)\right\| \leq & \| h\left(t, y_{t}+\bar{z}_{t}\right)+\int_{0}^{t}(t-s)^{\alpha-1} Q_{\alpha}(t-s) f\left(s, y_{t}+\bar{z}_{t} d s\right. \\
& -h\left(t, y_{t}+{\overline{z^{*}}}_{t}\right)-\int_{0}^{t}(t-s)^{\alpha-1} Q_{\alpha}(t-s) f\left(s, y_{t}+{\overline{z^{*}}}_{t}\right) d s \| \\
\leq & \left\|h\left(t, y_{t}+\bar{z}_{t}\right)-h\left(t, y_{t}+{\overline{z^{*}}}^{*}\right)\right\| \\
& \quad+\int_{0}^{t}(t-s)^{\alpha-1}\left\|Q_{\alpha}(t-s)\left(f\left(s, y_{t}+\bar{z}_{t}\right) d s-f\left(s, y_{t}+\overline{z^{*}} t\right)\right)\right\| d s \\
\leq & L_{h}\left\|z-z^{*}\right\|_{\mathfrak{B}}+\frac{\alpha M_{A}}{\Gamma(\alpha+1)} \int_{0}^{t}(t-s)^{\alpha-1} L_{f}\left\|z-z^{*}\right\|_{\mathfrak{B}} d s \\
\leq & \left.\left(C_{1} L_{h}+M_{A} C_{1} \frac{T^{\alpha} L_{f}}{\Gamma(\alpha+1)}\right)\right)\left\|z-z^{*}\right\|_{\mathfrak{B}^{\prime \prime}} .
\end{aligned}
$$

For $t \in\left[t_{i}, s_{i}\right] ; i=1,2, \ldots, m$

$$
\left\|P(z)(t)-P\left(z^{*}\right)(t)\right\| \leq\left(L_{I}+L_{g}\right)\left\|z-z^{*}\right\|_{\mathfrak{B}^{\prime \prime}},
$$

and for $t \in\left[s_{i}, t_{i+1}\right] ; i=1,2, \ldots, m$

$$
\begin{aligned}
& \left\|P(z)(t)-P\left(z^{*}\right)(t)\right\| \leq \| P_{\alpha}(t) d_{i}+h\left(t, y_{t}+\bar{z}_{t}\right)+\int_{0}^{t}(t-s)^{\alpha-1} Q_{\alpha}(t-s) f\left(s, y_{t}+\bar{z}_{t}\right) d s \\
& -P_{\alpha}(t) d_{i}^{*}+h\left(t, y_{t}+{\overline{z^{*}}}_{t}\right)-\int_{0}^{t}(t-s)^{\alpha-1} Q_{\alpha}(t-s) f\left(s, y_{t}+{\overline{z^{*}}}_{t}\right) d s \| \\
& \leq\left\|h\left(t, y_{t}+\bar{z}_{t}\right)-h\left(t, y_{t}+\overline{z^{*}} t\right)\right\|+\left\|P_{\alpha}(t) d_{i}-P_{\alpha}(t) d_{i}^{*}\right\| \\
& +\int_{0}^{t}(t-s)^{\alpha-1}\left\|Q_{\alpha}(t-s)\left(f\left(s, y_{t}+\bar{z}_{t}\right) d s-f\left(s, y_{t}+\overline{z^{*}} t\right)\right)\right\| d s \\
& \leq\left(M_{A}\left(L_{I}+L_{g}+C_{1} L_{h}+\frac{M_{A} C_{1} L_{f}}{\Gamma(\alpha+1)} T^{\alpha}\right)+\frac{C_{1} M_{A}}{\Gamma(\alpha+1)} T^{\alpha} C_{1} L_{f}+C_{1} L_{h}\right)\left\|z-z^{*}\right\|_{\mathfrak{B}^{\prime \prime}}
\end{aligned}
$$

where

$$
\begin{aligned}
& \left.d_{i}^{*}=I_{i}\left(y\left(t_{i}\right)+\overline{z^{*}}\left(t_{i}\right)\right)-h\left(s_{i}, y_{s_{i}}+\overline{z^{*} s_{i}}\right)\right)+g_{i}\left(s_{i}, y\left(s_{i}\right)+\overline{z^{*}}\left(s_{i}\right)\right) \\
& \left.-\int_{0}^{s_{i}}\left(s_{i}-s\right)^{\alpha-1} Q_{\alpha}\left(s_{i}-s\right) f\left(s, y_{s}+\overline{z^{*}}{ }_{s}\right)\right) d s .
\end{aligned}
$$

From what is mentioned above, for all $t \in[0, T]$, we deduce that

$$
\begin{aligned}
& \left\|P(z)(t)-P\left(z^{*}\right)(t)\right\|_{\mathfrak{B}^{\prime \prime}} \\
& \leq\left(M_{A}\left(L_{I}+L_{g}+C_{1} L_{h}+\frac{M_{A} C_{1} L_{f}}{\Gamma(\alpha+1)} T^{\alpha}\right)+\frac{C_{1} M_{A}}{\Gamma(\alpha+1)} T^{\alpha} C_{1} L_{f}+C_{1} L_{h}\right)\left\|z-z^{*}\right\|_{\mathfrak{B}^{\prime \prime}} .
\end{aligned}
$$

The operator $P$ is a contraction map on the space $\mathfrak{B}^{\prime \prime}$. Therefore it has a unique fixed point in $\mathfrak{B}^{\prime \prime}$, which is a mild solution of problem (1).

In the second part we use Lemma 2.2 to prove the existence of solution, but before that, we make the following hypothesis:

- $(H 5)$ The functions $I_{i}: X \rightarrow X$ are continuous and there exists a constant $M_{I}$ such that $\left\|I_{i}(x)\right\| \leq M_{I}, i=$ $1,2, \ldots, m$, for $x \in X$.

The functions $g_{i}:\left(t_{i}, s_{i}\right] \times X \rightarrow X$ are continuous and there exists a constant $M_{g}$ such that $\left\|g_{i}(t, x)\right\| \leq$ $M_{g}, i=1,2, \ldots, m$, for $x \in X$.

- (H6) $f$ and $h$ are continuous functions, and there exists a constant $M_{h}$ such that $\|h(t, x)\| \leq M_{h}$ for $t \in J$ and $x \in \mathfrak{B}$. 
- (H7) There exist $p, q \in C\left(J, \mathbb{R}^{+}\right)$, such that $\|f(t, x)\| \leq p(t)+q(t)\|x\|_{\mathfrak{B}}$ for $t \in J$ and $x \in \mathfrak{B}$.

Theorem 3.2. Assume that the assumptions, $(A),(H 2)$, and $(H 5)-(H 7)$ are satisfied. Then, the problem $(1)$ has at least one mild solution in $(-\infty, T]$.

Proof. We define again the operator $P$ as in Theorem 3.1, and we divide the proof into four steps:

- Step 1: $P$ is continuous.

Let $\left(z_{n}\right)_{n}$ be a sequence in $\mathfrak{B}^{\prime \prime}$, such that $z_{n} \rightarrow z$ in $\mathfrak{B}^{\prime \prime}$, then we have for $t \in\left[0, t_{1}\right]$

$$
\begin{aligned}
\left\|P\left(z_{n}\right)(t)-P(z)(t)\right\| \leq & \left\|h\left(t, \bar{z}_{n_{t}}+y_{t}\right)-h\left(t, \bar{z}_{t}+y_{t}\right)\right\| \\
& +\frac{\alpha M_{A}}{\Gamma(\alpha+1)} \int_{0}^{t}(t-s)^{\alpha-1}\left\|f\left(s, \bar{z}_{n_{s}}+y_{s}\right)-f\left(s, \bar{z}_{s}+y_{s}\right)\right\| d s .
\end{aligned}
$$

Since $f$ and $h$ are continuous functions, we have $\left\|P\left(z_{n}\right)(t)-P(z)(t)\right\|_{\mathfrak{B}^{\prime \prime}} \longrightarrow 0$ as $n \longrightarrow \infty$.

For $t \in\left[t_{i}, s_{i}\right], i=1,2, \ldots, m$

$$
\left.\| P\left(z_{n}\right)(t)-P(z)(t)\right)\|\leq\| I_{i}\left(z_{n}\left(t_{i}\right)\right)-I_{i}\left(z\left(t_{i}\right)\right)\|+\| g_{i}\left(z_{n}(t)\right)-g_{i}(z(t)) \|,
$$

Since the functions $I_{i}$ and $g_{i}$ are continuous for $i=1, \ldots, m$, we get

$$
\left.\| P\left(z_{n}\right)(t)-P(z)(t)\right) \|_{\mathfrak{B}^{\prime \prime}} \longrightarrow 0 \text { as } n \longrightarrow \infty .
$$

For $t \in\left[s_{i}, t_{i+1}\right] ;$ for $i=1,2, \ldots, m$

$$
\begin{aligned}
\left\|P\left(z_{n}\right)(t)-P(z)(t)\right\| \leq & \left\|h\left(t, \bar{z}_{n_{t}}+y_{t}\right)-h\left(t, \bar{z}_{t}+y_{t}\right)\right\| \\
& +\frac{\alpha M_{A}}{\Gamma(\alpha+1)} \int_{0}^{t}(t-s)^{\alpha-1}\left\|f\left(s, \bar{z}_{n_{s}}+y_{s}\right)-f\left(s, \bar{z}_{s}+y_{s}\right)\right\| d s \\
& +M_{A}\left(\left\|h\left(s_{i}, \bar{z}_{n_{s_{i}}}+y_{s_{i}}\right)-h\left(s_{i}, \bar{z}_{s_{i}}+y_{s_{i}}\right)\right\|\right. \\
& +\left\|I_{i}\left(z_{n}\left(t_{i}\right)\right)-I_{i}\left(z\left(t_{i}\right)\right)\right\|+\left\|g_{i}\left(z_{n}\left(s_{i}\right)\right)-g_{i}\left(z\left(s_{i}\right)\right)\right\| \\
& \left.+\frac{\alpha M_{A}}{\Gamma(\alpha+1)} \int_{0}^{s_{i}}\left(s_{i}-s\right)^{\alpha-1}\left\|f\left(s, \bar{z}_{n_{s}}+y_{s}\right)-f\left(s, \bar{z}_{s}+y_{s}\right)\right\| d s\right) .
\end{aligned}
$$

So

$$
\left\|P\left(z_{n}\right)(t)-P(z)(t)\right\|_{\mathfrak{B}^{\prime \prime}} \longrightarrow 0 \text { as } n \longrightarrow \infty .
$$

This implies that $P$ is continuous.

- Step 2: $P$ maps bounded sets into bounded sets in $\mathfrak{B}^{\prime \prime}$.

Indeed, we must prove that for $r>0$, there exists a positive $l$ such that for each $z \in B_{r}=\left\{z \in \mathfrak{B}^{\prime \prime},\|z\|_{\mathfrak{B}^{\prime \prime}} \leq r\right\}$ we have $\|P z\|_{\mathfrak{B}^{\prime \prime}} \leq l$. Let $z \in B_{r}$, 
for $t \in\left[0, t_{1}\right]$ we have

$$
\begin{aligned}
& \|P(z)(t)\|=\| P_{\alpha}(t)(\phi(0)-h(0, \phi))+h\left(t, y_{t}+\bar{z}_{t}\right)+ \\
& \qquad \int_{0}^{t}(t-s)^{\alpha-1} Q_{\alpha}(t-s) f\left(s, y_{s}+\bar{z}_{s}\right) d s \| \\
& \leq\left\|P_{\alpha}(t)(\phi(0)-h(0, \phi))\right\|+\left\|h\left(t, y_{t}+\bar{z}_{t}\right)\right\|+\frac{\alpha M_{A}}{\Gamma(\alpha+1)} \int_{0}^{t}(t-s)^{\alpha-1}\left\|f\left(s, y_{s}+\bar{z}_{s}\right)\right\| d s \\
& \left.\leq M_{A}\left(\|\phi(0)\|+M_{h}\right)+M_{h}+\frac{T^{\alpha} M_{A}}{\Gamma(\alpha+1)}\left(\|p\|_{\infty}+\|q\|_{\infty} \| y_{s}+\bar{z}_{s}\right) \|_{\mathfrak{B}}\right) \\
& \left.\leq M_{A}\left(\|\phi(0)\|+M_{h}\right)+M_{h}+\frac{T^{\alpha} M_{A}}{\Gamma(\alpha+1)}\left(\|p\|_{\infty}+\|q\|_{\infty}\left(\left\|y_{s}\right\|_{\mathfrak{B}}+\| \bar{z}_{s}\right) \|_{\mathfrak{B}}\right)\right) \\
& \leq M_{A}\left(\|\phi(0)\|+M_{h}\right)+M_{h}+\frac{T^{\alpha} M_{A}}{\Gamma(\alpha+1)}\left(\|p\|_{\infty}+\|q\|_{\infty}\left(C_{2}\|\phi\|_{\mathfrak{B}}+C_{1} r\right)\right) \\
& \left.\leq M_{A}\left(\|\phi(0)\|+M_{h}\right)+\frac{T^{\alpha} M_{A}}{\Gamma(\alpha+1)}\left(\|p\|_{\infty}+\|q\|_{\infty} r^{*}\right)\right)+M_{h},
\end{aligned}
$$

with $r^{*}=C_{2}\|\phi\|_{\mathfrak{B}}+C_{1} r$.

For $t \in\left[t_{i}, s_{i}\right], i=1,2, \ldots, m$

$$
\begin{aligned}
\|P(z)(t)\| & \leq\left\|I_{i}\left(z\left(t_{i}\right)\right)\right\|+\| g_{i}(t, z(t) \| \\
& \leq M_{I}+M_{g}=l_{2}
\end{aligned}
$$

For $t \in\left[s_{i}, t_{i+1}\right] ; i=1,2, \ldots, m$

$$
\begin{aligned}
\|P(z)(t)\|= & \left\|P_{\alpha}(t) d_{i}+h\left(t, y_{t}+\bar{z}_{t}\right)+\int_{0}^{t}(t-s)^{\alpha-1} Q_{\alpha}(t-s) f\left(s, y_{s}+\bar{z}_{s}\right) d s\right\| \\
\leq & \left.\left\|P_{\alpha}(t) d_{i}\right\|+M_{h}+\frac{T^{\alpha} M_{A}}{\Gamma(\alpha+1)}\left(\|p\|_{\infty}+\|q\|_{\infty} r^{*}\right)\right) \\
\leq & M_{A}\left(M_{I}+M_{g}+M_{h}+M_{A}\left(\frac{T^{\alpha}}{\Gamma(\alpha+1)}\left(\|p\|_{\infty}+\|q\|_{\infty} r^{*}\right)\right)\right. \\
& +M_{h}+\frac{T^{\alpha} M_{A}}{\Gamma(\alpha+1)}\left(\|p\|_{\infty}+\|q\|_{\infty} r^{*}\right) \\
\leq & M_{A}\left(M_{I}+M_{g}+M_{h}+\left(1+M_{A}\right) \frac{T^{\alpha}}{\Gamma(\alpha+1)}\left(\|p\|_{\infty}+\|q\|_{\infty} r^{*}\right)\right)+M_{h}=l_{3} .
\end{aligned}
$$

This implies that $\|P z\|_{\mathfrak{B}^{\prime \prime}} \leq \max \left(l_{1}, l_{2}, l_{3}\right)=l$, for $t \in[0, T]$.

- Step 3: $P$ maps bounded sets into equi-continuous sets of $\mathcal{B}^{\prime \prime}$.

Let $B_{r}$ be the set of step 2 . Then, for every $t^{\prime}, t^{\prime \prime} \in\left[0, t_{1}\right]$, with $t^{\prime}<t^{\prime \prime}$ and $z \in B_{r}$, we have

$$
\begin{aligned}
\left\|P(z)\left(t^{\prime \prime}\right)-P(z)\left(t^{\prime}\right)\right\| & \leq\left\|\left(P_{\alpha}\left(t^{\prime \prime}\right)-P_{\alpha}\left(t^{\prime}\right)\right)(\phi(0)+h(0, \phi))\right\| \\
& +\left\|h\left(t^{\prime}, y_{t^{\prime}}+\bar{z}_{t^{\prime}}\right)-h\left(t^{\prime \prime}, y_{t^{\prime \prime}}+\bar{z}_{t^{\prime \prime}}\right)\right\|+E_{1}+E_{2}+E_{3},
\end{aligned}
$$

where

$$
\begin{gathered}
\left.E_{1}=\| \int_{t^{\prime}}^{t^{\prime \prime}}\left(t^{\prime \prime}-s\right)^{\alpha-1} Q_{\alpha}\left(t^{\prime \prime}-s\right) f\left(s, y_{s}+\bar{z}_{s}\right)\right) d s \|, \\
\left.E_{2}=\| \int_{0}^{t^{\prime}}\left(\left(t^{\prime \prime}-s\right)^{\alpha-1}-\left(t^{\prime}-s\right)^{\alpha-1}\right) Q_{\alpha}\left(t^{\prime \prime}-s\right) f\left(s, y_{s}+\bar{z}_{s}\right)\right) d s \|, \\
\left.E_{3}=\| \int_{0}^{t^{\prime}}\left(t^{\prime}-s\right)^{\alpha-1}\left(Q_{\alpha}\left(t^{\prime \prime}-s\right)-Q_{\alpha}\left(t^{\prime}-s\right)\right) f\left(s, y_{s}+\bar{z}_{s}\right)\right) d s \| .
\end{gathered}
$$

Following the idea in [13, 14, we can prove that $E_{1}, E_{2}, E_{3}$ tend to zero as $t^{\prime} \longrightarrow t^{\prime \prime}$. Since $P_{\alpha}(t)$ and $P_{\alpha}(t)$ $(t>0)$ are continuous in $t$, in the uniform operator topology, 
we deduce that $\left\|P(z)\left(t^{\prime \prime}\right)-P(z)\left(t^{\prime}\right)\right\| \longrightarrow 0$ as $t^{\prime} \longrightarrow t^{\prime \prime}$.

For every $t^{\prime}, t^{\prime \prime} \in\left[t_{i}, s_{i}\right]$, with $t^{\prime}<t^{\prime \prime}$, we have

$$
\left\|P(z)\left(t^{\prime \prime}\right)-P(z)\left(t^{\prime}\right)\right\| \leq\left\|g_{i}\left(t^{\prime \prime}, y\left(t^{\prime \prime}\right)+\bar{z}\left(t^{\prime \prime}\right)\right)-g_{i}\left(t^{\prime}, y\left(t^{\prime}\right)+\bar{z}\left(t^{\prime}\right)\right)\right\| .
$$

Since the functions $g_{i}$ are continuous, for $i=1,2, \ldots, m$, then $\left\|P(z)\left(t^{\prime \prime}\right)-P(z)\left(t^{\prime}\right)\right\| \longrightarrow 0$ as $t^{\prime \prime} \longrightarrow t^{\prime}$.

And for every $t^{\prime}, t^{\prime \prime} \in\left[t_{i}, s_{i}\right]$, such that $t^{\prime}<t^{\prime \prime}$ then

$$
\begin{aligned}
\left\|P(z)\left(t^{\prime \prime}\right)-P(z)\left(t^{\prime}\right)\right\| & \left.\leq \|\left(P_{\alpha}\left(t^{\prime \prime}\right)-P_{\alpha}\left(t^{\prime}\right)\right) d_{i}\right) \| \\
& +\left\|h\left(t^{\prime}, y_{t^{\prime}}+\bar{z}_{t^{\prime}}\right)-h\left(t^{\prime \prime}, y_{t^{\prime \prime}}+\bar{z}_{t^{\prime \prime}}\right)\right\|+E_{1}+E_{2}+E_{3} .
\end{aligned}
$$

Similarly to the case where $t \in\left[0, t_{1}\right]$, we prove that $\left\|P(z)\left(t^{\prime \prime}\right)-P(z)\left(t^{\prime}\right)\right\| \longrightarrow 0$ as $t^{\prime \prime} \longrightarrow t^{\prime}$.

- Step 4: We show that the set $\Omega=\left\{z \in \mathcal{B}^{\prime \prime}: z=\lambda P z\right\}$ is bounded.

Let $z \in \Omega$, then $z(t)=\lambda P z(t)$, with $0<\lambda<1$. For $t \in\left[0, t_{1}\right]$, we have

$$
\begin{aligned}
\|z(t)\| \leq & \lambda\left(\|\left(P_{\alpha}(t)(\phi(0)-h(0, \phi))+h\left(t, y_{t}+\bar{z}_{t}\right)\right.\right. \\
& \left.\left.+\int_{0}^{t}(t-s)^{\alpha-1} Q_{\alpha}(t-s) f\left(s, y_{s}+\bar{z}_{s}\right) d s\right) \|\right) \\
\leq & \lambda\left(M_{A}\left(\|\phi\|+M_{h}\right)+M_{h}+\frac{\alpha M_{A}}{\Gamma(\alpha+1)}\left(\int_{0}^{t}(t-s)^{\alpha-1}\left\|f\left(s, y_{s}+\bar{z}_{s}\right)\right\| d s\right)\right) .
\end{aligned}
$$

For $t \in\left[t_{i}, s_{i}\right]$, we have for $i=1,2, \ldots, m$,

$$
\begin{aligned}
\|z(t)\| & \leq\left\|\lambda I_{i}\left(y\left(t_{i}\right)+\bar{z}\left(t_{i}\right)\right)\right\|+\| \lambda g_{i}(t, y(t)+\bar{z}(t) \| \\
& \leq \lambda\left(M_{I}+M_{g}\right) .
\end{aligned}
$$

For $t \in\left[s_{i}, t_{i+1}\right]$, for $i=1,2, \ldots, m$, we have

$$
\begin{aligned}
\|z(t)\| & =\left\|P_{\alpha}(t) d_{i}+h\left(t, y_{t}+\bar{z}_{t}\right)+\int_{0}^{t}(t-s)^{\alpha-1} Q_{\alpha}(t-s) f\left(s, y_{s}+\bar{z}_{s}\right) d s\right\| \\
& \leq M_{A}\left\|d_{i}\right\|+\|h\|+\frac{\alpha M_{A}}{\Gamma(\alpha+1)} \int_{0}^{t}(t-s)^{\alpha-1}\left\|f\left(s, y_{s}+\bar{z}_{s}\right)\right\| d s,
\end{aligned}
$$

then for $t \in[0, T]$, we have

$$
\begin{aligned}
\|z(t)\| \leq & M_{A} D+M_{h}+\frac{\alpha M_{A}}{\Gamma(\alpha+1)} \int_{0}^{t}(t-s)^{\alpha-1}\left\|f\left(s, y_{s}+\bar{z}_{s}\right)\right\| d s \\
\leq & M_{A} D+M_{h}+\frac{\alpha M_{A}}{\Gamma(\alpha+1)} \int_{0}^{t}(t-s)^{\alpha-1}\left(p(s)+q(s)\left\|y_{s}+\bar{z}_{s}\right\|\right) d s \\
\leq & M_{A} D+M_{h}+\frac{T^{\alpha} M_{A}\left(P_{1}+q_{1} C_{2}\|\phi\|\right)}{\Gamma(\alpha+1)} \\
& +\frac{\alpha C_{1} q_{2} M_{A}}{\Gamma(\alpha+1)} \int_{0}^{t}(t-s)^{\alpha-1} \sup _{0 \leq \tau \leq s}\|z(\tau)\| d s \\
\leq & W_{1}+W_{2} \int_{0}^{t}(t-s)^{\alpha-1} \sup _{0 \leq \tau \leq s}\|z(\tau)\| d s
\end{aligned}
$$


where

$$
\begin{gathered}
D=\max _{1 \leq i \leq m} d_{i}, \quad W_{1}=M_{A}\left\|d_{i}\right\|+M_{h}+\frac{T^{\alpha} M_{A}\left(P_{1}+q_{1} C_{2}\|\phi\|\right)}{\Gamma(\alpha+1)}, \\
W_{2}=\frac{\alpha C_{1} q_{2} M_{A}}{\Gamma(\alpha+1)}, \quad p_{1}=\sup _{t \in[0, T]} p(t), \quad q_{1}=\sup _{t \in[0, T]} q(t) .
\end{gathered}
$$

Let $\tau^{*} \in[0, s]$ be such that $\sup _{0 \leq \tau \leq s}\|z(\tau)\|=\left\|z\left(\tau^{*}\right)\right\|, \quad 0 \leq s \leq t$, and we use Lemma 2.3, then there exists a constant $K(\alpha)$ such that

$$
\begin{aligned}
\|z(t)\| & \leq W_{1}+K(\alpha) W_{1} \int_{0}^{t}(t-s)^{\alpha-1} d s \\
& \leq W_{1}\left(1+\frac{K(\alpha) T^{\alpha}}{\alpha}\right) .
\end{aligned}
$$

Using lemma 2.4, we deduce that $P$ has at least a fixed point on $(-\infty, T]$. This completes the proof of theorem.

Acknowledgements. The authors would like to thank the referees for their valuable suggestions and comments.

\section{References}

[1] M. Benchohra, J.Henderson, S. K. Ntoyas, and A. Ouahab, Existence results for fractional order functional differential equations with infinite delay, J. Math. Anal. Appl. 338:1340-1350, 2008.

[2] J. Dabas, A. Chauhan, and M. Kumar, Existence of the Mild Solutions for Impulsive Fractional Equations with Infinite Delay, International Journal of Differential Equations, Volume 2011, doi: 10.1155/2011/793023.

[3] X. Fu, X. Liu and B. Lu, On a new class of impulsive fractional evolution equations, Advances in Difference Equations, 2015:227, 2015.

[4] J. Hale and J.Kato, Phase space for retarded equations with infinite delay, Funkcial. Ekvac. 21:11-41, 1978.

[5] H. Jiang, Existence results for fractional order functional differential equations with impulse, Comput. Math. Appl., 64:3477-3483, 2012.

[6] A. A. Kilbas, H. M. Srivastava, and J. J. Trujillo, Theory and Applications of Fractional Differential Equations, in: North-Holland Mathematics Studies, vol. 204, Elsevier, Amsterdam, 2006.

[7] N. Kosmatov, Integral equations and initial value problems for nonlinear differential equations of fractional order, Nonlinear Anal. TMA, 70:2521-2529, 2009.

[8] V. Lakshmikantham, Theory of fractional functional differential equations, Nonlinear Anal. TMA, 69(10):33373343,2008 .

[9] V. Lakshmikantham and A.S. Vatsala, Basic theory of fractional differential equations, Nonlinear Anal. 69(8):2677-2682, 2007.

[10] K.S. Miller and B. Ross, An Introduction to the Fractional Calculus and Fractional Differential Equations, Wiley, New York, 1993.

[11] I. Podlubny, Fractional Differential Equations, Academic Press, New York, 1993.

[12] Y. Zhou, F. Jiao, and J. Li, Existence and uniqueness for fractional neutral differential equations with infinite delay, Nonlinear Anal. TMA, 71:3249-3256, 2009.

[13] Y. Zhou, Basic theory of fractional differential equations, Xiangtan University, China, 2014.

[14] Y. Zhou and F. Jiao, Existence of mild solutions for fractional neutral evolution equations, Comput. Math. Appl., 59:1063-1077, 2010. 